\title{
A Universal Model of Spin Relaxation
}

\section{A first-principles theory predicts nonequilibrium spin dynamics, including various quantum scatterings for general solid materials.}

\author{
By Rachel Berkowitz
}

$\square$ pintronic devices and quantum-information applications that use spin qubits require materials that can sustain spin coherence for long periods at room temperature. First-principles predictions of spin lifetimes have been limited to materials with certain crystal symmetries, or they have required that the system begins from a quasiequilibrium state. Yuan Ping at the University of California, Santa Cruz, and her colleagues now provide computational tools that accurately predict spin lifetime for any material-even those that are out of equilibrium [1]. By capturing contributions to the spin dynamics that have not been observed using previous methods, their approach can determine the dominant process that leads to decoherence.

Ping's team recently tackled the crystal-symmetry limitation by developing a generalized rate equation for spin relaxation due to spin-phonon interaction in systems near equilibrium. Their new study extends that framework to encompass open quantum systems and the scattering processes-including
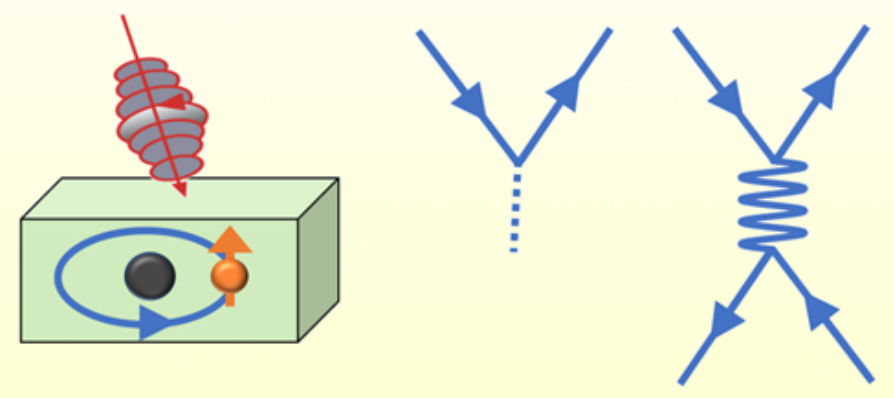

electron-phonon, electron-impurity, and electron-electron interactions - that result from ultrafast pump-probe measurement techniques. The researchers used this extended framework to model spin behavior in gallium arsenide, a common semiconductor material for spintronics. They compared simulations of ultrafast measurements to experimental values of coupled spin and electron dynamics for gallium arsenide at different temperatures and doping levels. They also examined ultrafast dynamics in one- and two-layer transition-metal dichalcogenides, which are important materials for a new type of quantum technology called valleytronics.

In all cases, the simulations proved accurate down to submicrosecond timescales, allowing the team to pick out which scattering pathway dominated the spin-relaxation process. By providing such information for any solid system, the new computational platform promises fundamental insights into spin relaxation, which, the researchers say, is critical for the design of new quantum materials.

Rachel Berkowitz is a Corresponding Editor for Physics based in Vancouver, Canada.

\section{REFERENCES}

1. J. Xu et al., "Ab initio ultrafast spin dynamics in solids," Phys. Rev. B 104, 184418 (2021).

Credit: J. Xu/University of California, Santa Cruz 